\title{
Frequency Agile Radial-Shaped Varactor-Loaded Reflectarray Cell
}

\author{
Francesca VENNERI, Sandra COSTANZO, Giuseppe DI MASSA, Antonio BORGIA, Antonio RAFFO \\ DIMES, University of Calabria, Via P. Bucci cubo 42C, 87036 Rende (CS), Italy
}

costanzo@dimes.unical.it

Manuscript received December 07, 2015

\begin{abstract}
An equivalent circuit approach is adopted in this paper to analyze a novel varactor loaded phasing line, specifically designed to improve the frequency agility features of reconfigurable aperture-coupled reflectarray cell, through the use of a couple of microstrip radial stubs. The proposed analysis method is fruitfully implemented to perform a fast and preliminary investigation on the improvements provided by the radial shaped phasing line in terms of frequency agility of the reflectarray unit cell. The method is adopted to compare frequency performances of radial and linear phasing line geometries, allowing to effectively demonstrate the radial line geometry contribution to the enhancement of the unit cell frequency performances.
\end{abstract}

\section{Keywords}

Microstrip radial line, varactor, reflectarray

\section{Introduction}

Active reflectarrays give a promising and attractive solution for the implementation of modern reconfigurable antenna systems, being able to offer different reconfiguration functions, including beam-scanning and radiation pattern reshaping, frequency and polarization agility, strongly required by a large number of RF applications, such as radars and satellite communications. Several recent papers [1-4] demonstrate the effectiveness of reconfigurable reflectarrays in replacing mechanically moved reflectors or phased arrays, leading many other benefits strictly related to reflectarray concept, such as low profile, simpler architectures, increased efficiencies, instantaneous radar beam positioning [5], [6], and absence of mechanical vibrations. Furthermore, active reflectarrays allow to simultaneously achieve one or more reconfiguration functionalities, by simply acting at the unit cell level, to have reconfigurable elements integrated with more electronically tunable components. For example, many reflectarray configurations, among those described in [2], [3], offer polarization flexibility, frequency agility or multi-band operation through the use/integration of one or more active compo- nents, such as MEMS and PIN/varactor diodes. A dual polarized reflectarray cell loaded by two varactor pairs has been proposed in [7], while a first proof-of-concept of multi-band reflectarray has been reported in [8]. A reflectarray element based on the use of a couple of PIN-diodes and a varactor has been presented in [9], to tune the element reflection phase within a wide frequency range.

A promising and versatile reconfigurable reflectarray cell has been proposed by the authors in [10-13]. It consists of a rectangular patch aperture-coupled to a uniform microstrip line loaded by a single varactor/varicap diode. By applying a tunable reverse DC voltage across the varicap, the diode capacitance can be properly tuned in order to actively adjust the reflection phase of the single element, thus providing a dynamic control of the backscattering features relative to the elementary cell. This latter has been experimentally validated in [13], by demonstrating good beam steering as well as pattern reshaping capabilities. The above configuration has been further modified in [14], to improve the unit cell frequency performances in terms of frequency agility. In particular, by adopting a couple of radial lines to replace the linear stubs used in [13], the unit cell operational bandwidth (i.e. the frequency range within which it is possible to effectively tune the unit cell reflection phase in a quite full phase-range) is roughly tripled, thus achieving a good degree of frequency agility. Practically, the structure proposed in [14] exploits the broadband behavior of the radial line geometry [15] to extend the unit cell reconfiguration capabilities over a wider frequency range, without adding any further tunable components.

In this paper, an equivalent circuit-model approach is adopted to analyze the wider band behavior of the radial phasing line configuration [14] with respect to the linear one [13]. The adopted analysis method is fruitfully implemented to perform a fast and preliminary investigation on the improvements provided by the radial shaped varactor loaded phasing line in terms of reflectarray unit cell frequency agility. A $10 \mathrm{GHz}$ test case is extensively analyzed to show the accuracy of the proposed simplified analysis approach in demonstrating the useful radial line contribution in the enhancement of the reflectarray unit cell frequency performances, at very low computational cost. 


\section{Operational Bandwidth Evaluation of Varactor Loaded Unit Cells}

The active reflectarray unit cells assumed in this work are illustrated in Fig. 1. Both elements have been designed and proposed by the authors in some previous papers [13], [14]. In particular, the configuration depicted in Fig. 1(a) has been experimentally tested in [13], [16], while the cell illustrated in Fig. 1(b) has been proposed in [14] as an improved version of the former, to enhance the unit cell frequency performances. The basic structure, common to both cells, consists of a rectangular patch aperture-coupled to a microstrip phasing line. This is loaded by a single varactor diode, whose tunable capacitance allows to achieve a dynamic control of the phase response relative to the unit cells. In particular, as discussed in [13], [14], the stubs of each configuration (Figs. 1(a), 1(b)) are properly sized to achieve a reconfigurable coupled line that behaves like a tunable LC circuit, thus acting as a $360^{\circ}$ phase shifter. Moreover, the patch and the slot sizes are chosen to simultaneously obtain antenna resonance condition and impedance matching between patch and coupled phasing line.

The main difference between the two considered cells lies in the phasing line geometry. As a matter of fact, the first cell depicted in Fig. 1(a) is electronically driven by a uniform microstrip line composed by two linear segments $L_{\mathrm{s}}$ and $L_{\mathrm{v}}$, while the second cell (Fig. 1(b)) is tuned by a phasing line consisting of two radial stubs, characterized by a $90^{\circ}$-aperture angle and radius respectively equal to $r_{1}$ and $r_{2}$. The results achieved in [14] demonstrate how the last phasing line configuration allows to improve the frequency performances of the unit cell, thanks to the wideband behavior of the radial stubs. In fact, as demonstrated in [14], a radial shaped microstrip stub is typically characterized by an input impedance having slower changes versus frequency, if compared with the reactance of an equivalent linear stub.

A case study extracted from [14] is reported in the following, demonstrating radial stub potentials in designing frequency agile reflectarray cells. Figure 2 illustrates the comparison between the reflection phase curves versus frequency computed for different varactor capacitances, both for a $10 \mathrm{GHz}$-cell loaded by a radial phasing line (Fig. 1(b)) as well as for an equivalent one tuned by a linear phasing line (Fig. 1(a)). Either cells are characterized by sizes equal to $0.45 \lambda_{0} \times 0.45 \lambda_{0}$ and the layers stratification (Fig. 1(c)) reported in Tab. 1. A varactor diode having a capacitance varying from $0.2 \mathrm{pF}$ up to $2 \mathrm{pF}$ is considered to dynamically control the phase response of the unit cells. Both phasing lines are properly sized to achieve the following values: $L_{\mathrm{s}}=6 \mathrm{~mm}, L_{\mathrm{v}}=4.6 \mathrm{~mm}$ (Fig. 1(a)) and $r_{1}=4.3 \mathrm{~mm}, r_{2}=2.7 \mathrm{~mm}$ (Fig. 1(b). The above dimensions are computed to obtain an alternate capacitive-inductive behavior in the phase of the signal reflected towards the aperture-coupled patch (Fig. 1), by varying the diode capacitance within the available tuning range (see [14] for

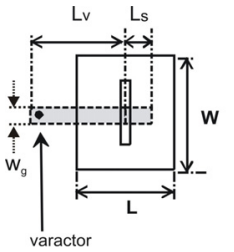

(a)

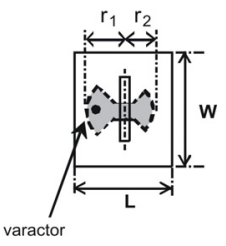

(b)

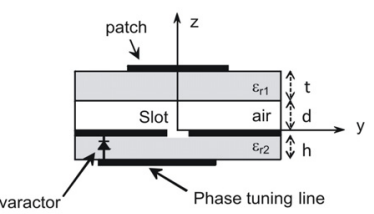

(c)

Fig. 1. Reflectarray element layout : (a) aperture-coupled cell with linear phasing line; (b) aperture-coupled cell with radial phasing line; (c) unit cell layers stratification.

\begin{tabular}{|c|c|c|}
\hline Layer & Material & Thickness \\
\hline Patch & Copper & $35 \mu \mathrm{m}$ \\
\hline Antenna substrate & Diclad870 $\left(\varepsilon_{\mathrm{r} 1}=2.33\right)$ & $t=0.762 \mathrm{~mm}$ \\
& Air & $d=1.524 \mathrm{~mm}$ \\
\hline Ground plane with slot & Copper & $35 \mu \mathrm{m}$ \\
\hline Phasing line substrate & TC600 $\left(\varepsilon_{\mathrm{r} 2}=6.15\right)$ & $h=0.762 \mathrm{~mm}$ \\
\hline Phasing line & Copper & $35 \mu \mathrm{m}$ \\
\hline
\end{tabular}

Tab. 1. Element layers stratification.

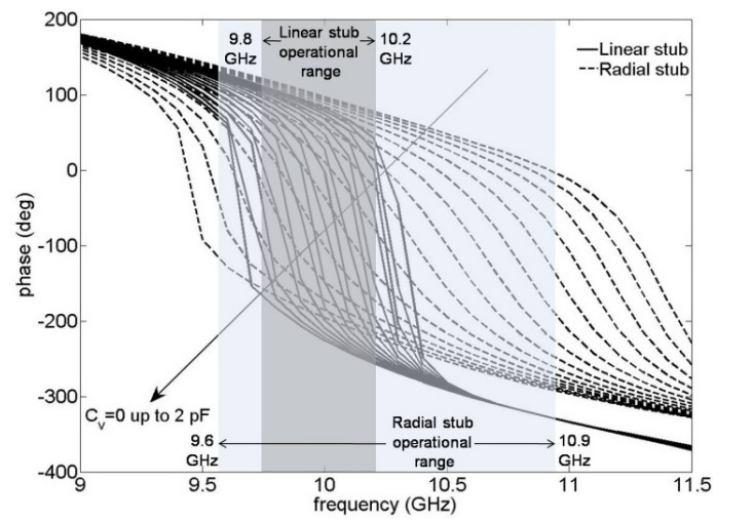

Fig. 2. Simulated reflection phase vs. frequency for different varactor capacitance $C_{\mathrm{v}}$ : comparison between cells driven by linear and radial phasing lines.

details). Moreover, the patch and the slot sizes are chosen to simultaneously obtain the antenna resonance and the matching with the phasing line, achieving in both cases the following dimensions (Fig. 1): $L=8.9 \mathrm{~mm}, \mathrm{~W}=6.8 \mathrm{~mm}$, $\mathrm{L}_{\mathrm{a}}=6.7 \mathrm{~mm}, \mathrm{~W}_{\mathrm{a}}=0.7 \mathrm{~mm}$. The above unit cells are analyzed through the use of the infinite array approach, assuming a normally incident plane wave.

The phase curves depicted in Fig. 2 show that the unit cell operational bandwidth (i.e. the frequency range within which it is possible to effectively tune the unit cell reflection phase in a full phase-range greater than $300^{\circ}$ ) increases threefold when the radial shaped phasing line is adopted instead of the classic linear one. These results confirm the possibility to design an aperture-coupled cell with a certain degree of frequency agility, through a proper shaping of the tuning line, without adding any further active components. 


\section{Numerical Modeling of Varactor Loaded Radial Phasing Line}

As pointed out in the above paragraph, the slower frequency variations of the input impedance relative to the radial stubs, are fruitfully exploited in [14] to design a novel broadband varactor loaded phasing line, suitable to improve the frequency performances of the aperture-coupled reflectarray cell proposed in [13].

In order to better justify the above results, a preliminary numerical characterization of the adopted radial phasing line geometry is performed in this paragraph. Using the equivalent-circuit model approach adopted in [13] to analyze linear phasing line performances, which is reported under Fig. 3(a), the reflection phase behavior at section AA', corresponding to the slot center, is evaluated through the use of the circuit model illustrated in Fig. 3(b). The line input impedance $Z_{\mathrm{AA}}$, , seen by the slot (Fig. 3(b)), is computed as the series combination of the two radial stubs impedances, which in turns are computed through the adoption of the simplified numerical approach proposed in [17]. This last method models the radial microstrip line with a set of cascaded interconnections of uniform transmission lines [18] having equal incremental length $\mathrm{d} l$ and width $w_{k}$ fixed by the radial shape (Fig. 3(c)).

The input impedance of each radial stub is recursively calculated through the following equation:

$$
Z_{\text {in }}^{(k)}=Z_{0 k} \frac{Z_{\text {in }}^{(k+1)}+j Z_{0 k} \tan \beta_{k} \mathrm{~d} l}{Z_{0 k}+j Z_{\text {in }}^{(k+1)} \tan \beta_{k} \mathrm{~d} l}
$$

where $Z_{\text {in }}{ }^{(k)}$ is the input impedance of the $k^{\text {th }}$ cascaded uniform transmission line, $Z_{\text {in }}{ }^{(k+1)}$ is the load impedance of the $k^{\text {th }}$ line, that is equal to the input impedance of the $(k+1)^{\text {th }}$ section (Fig. 3(b)), while $Z_{0 k}$ and $\beta_{k}$ are the characteristic impedance and the phase constant of the $k^{\text {th }}$ transmission line segment, computed through the formulas reported in [19]. The recursive equation (1) is implemented starting from the line section placed at the end of each stub down to the input section having width $w_{\mathrm{g}}$ (Fig. 3(c)). In particular, the initial value of the recursive equation (1) is set equal to the varactor diode impedance $\left(Z_{\mathrm{var}}\right)$, in the case of stub $r_{1}$, and an open-circuit load for stub $r_{2}$ (Fig. 3(b)).

For the sake of simplicity, the following approximations are introduced: both dielectric and conductor losses are neglected, the frequency dependence of the line segments is not considered, the fringing effect is neglected, the electrical parameters of varactor diode, including package parasitic effects, are taken into account, but its physical size is neglected.

Even if more accurate methods can be adopted to estimate the input impedance of the radial stub and perform a more accurate line sizing (e.g. the closed-form formula derived in [15]), the above simplified approach is sufficient to get a rough idea on the radial stub input behavior, giving us a fast and useful tool to demonstrate the operational principle of varactor loaded radial phasing lines, which is
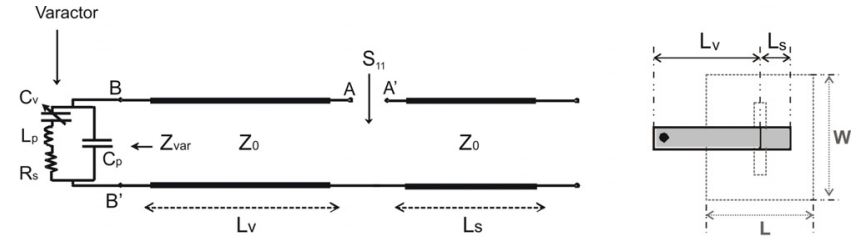

(a)
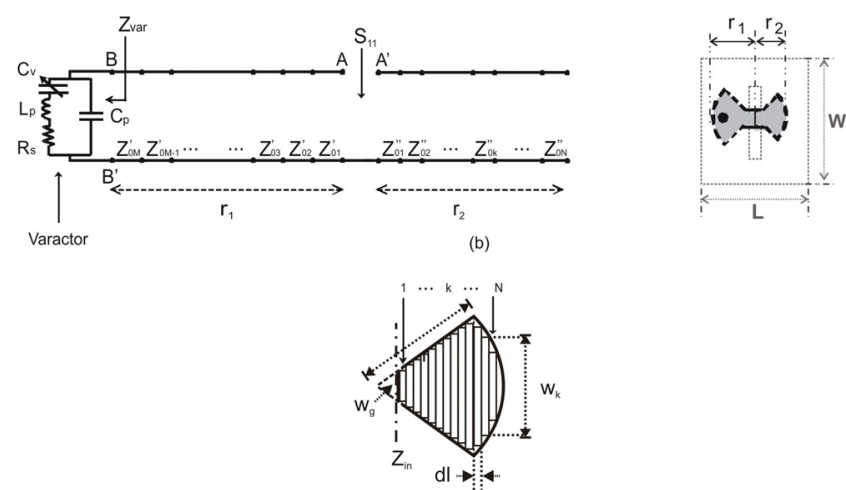

(c)

Fig. 3. Varactor loaded phasing line circuit model: (a) linear phasing line model; (b) radial phasing line model; (c) radial stub geometry approximation.

the main goal of this work, as well as to estimate an initial value for the radial stubs size. Furthermore, the proposed circuit model can take into account the variable capacitive load, simply by loading the $M^{\text {th }}$ line section at the end of stub $r_{1}$ with the varactor impedance $Z_{\text {var }}$ (Fig. 3(b)).

The above circuit model is adopted in this work to perform a preliminary analysis of the benefits offered by the radial phasing line geometry, in terms of reflection phase frequency stability, and to find out the motivations that lead to these improved frequency performances.

At this purpose, the radial phasing line is properly sized to achieve a fully tunable reflection phase at section AA' (i.e. $\left.\arg \left(S_{11}\right)\right)$, in correspondence of the design frequency $f=10 \mathrm{GHz}$. The input port width $w_{\mathrm{g}}$ is fixed to a value of $1.6 \mathrm{~mm}$, leading to a characteristic impedance equal to $Z_{0}^{\prime}=Z_{0}^{\prime \prime}=40 \Omega$, when the line is printed on a substrate having $\varepsilon_{\mathrm{r}}=6.15$ and thickness $h=0.762 \mathrm{~mm}$. The diode impedance $Z_{\mathrm{var}}$ is modeled with the equivalent circuit depicted in Fig. 3. This is composed by a tunable capacitance $C_{\mathrm{v}}$ varying from 0.2 up to $2 \mathrm{pF}$, a diode series resistance $R_{\mathrm{s}}=1.36 \Omega$, and package parasitic inductance/capacitance respectively equal to $L_{\mathrm{p}}=0.2 \mathrm{nH}$ and $C_{\mathrm{p}}=$ $0.15 \mathrm{pF}$ (typical values representing the Microsemi MV31011-89 diode model).

As graphically demonstrated in Figs. 4 and 5, the circuit model is firstly adopted to get out a proper sizing of the two radial branches composing the phasing line. The above mentioned figures illustrate, respectively, the reflection phase $\left(\arg \left(S_{11}\right)\right)$ and the input reactance $\left(\operatorname{Imag}\left(Z_{\mathrm{AA}^{\prime}}\right)\right)$ curves, computed by varying the diode capacitance from 0 up to $4 \mathrm{pF}$, for different lengths of the radial sections $r_{1}$ and $r_{2}$. For the sake of simplicity, only a significant set of curves is considered, corresponding to a couple of radial 


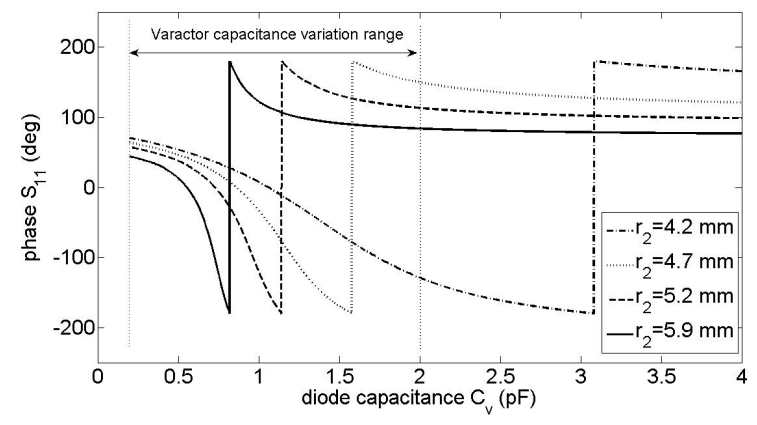

Fig. 4. Phase of coefficient $S_{11}$ versus diode capacitance computed for different values of $r_{2}\left(r_{1}=6.06 \mathrm{~mm}\right)$.

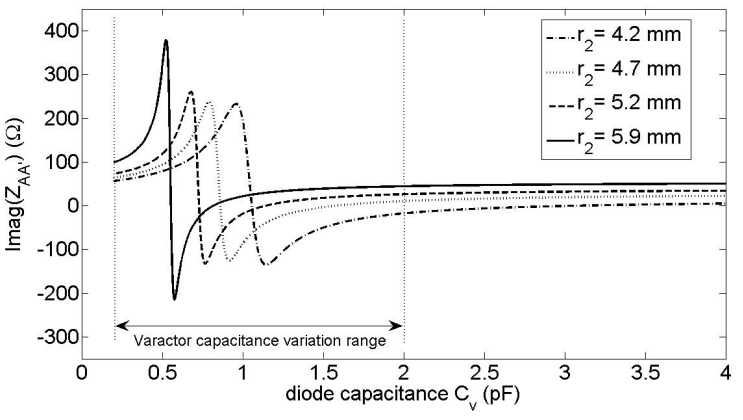

Fig. 5. Input reactance at port $\mathrm{AA}^{\prime}$ versus diode capacitance computed for different values of $r_{2}\left(r_{1}=6.06 \mathrm{~mm}\right)$.

stubs having the following lengths: $r_{1}$ equal to $6.06 \mathrm{~mm}$ and $r_{2}$ varying from $4.2 \mathrm{~mm}$ up to $5.9 \mathrm{~mm}$. From the curve reported in Fig. 4, it can be observed how a proper choice of stubs radius can optimize the variation interval of the reflection phase curve, also when the capacitance tuning range is fixed by the adopted diode model (in our case it is set to $[0.2 \div 2] \mathrm{pF}$ ). On the other side, Figure 5 demonstrates how the alternate capacitive-inductive behavior of the impedance $Z_{\mathrm{AA}}$, can be exploited to achieve a quite-full phase variation range.

Once chosen the stubs radii through the maximization of the $\mathrm{S}_{11}$-phase variation range against the diode capacitance $C_{\mathrm{v}}$ (i.e. $r_{1}=6.06 \mathrm{~mm}$ and $r_{2}=5.9 \mathrm{~mm}$ in Fig. 4), the proposed circuit model approach is adopted to evaluate the frequency behavior of the radial phasing line response. In other words, the phase of reflection coefficient $S_{11}$ is computed as a function of frequency, for each value of the diode capacitance $C_{\mathrm{v}}$ ranging from $0.2 \mathrm{pF}$ up to $2 \mathrm{pF}$. This last analysis gives the curves depicted in Fig. 6. A phase variation range greater than $300^{\circ}$ can be observed at all frequencies belonging to a quite large neighborhood around $10 \mathrm{GHz}$ (Fig. 6), thus defining the phasing line operational range. In the same figure, it can be appreciated how the above frequency interval results to be greater than that offered by an equivalent linear phasing line (Fig. 3(a)) printed on the same substrate and characterized by $L_{\mathrm{v}}=4.25 \mathrm{~mm}$ and $L_{\mathrm{s}}=6.5 \mathrm{~mm}$.

The above result can be attributed to the slower frequency variations of the input impedance $Z_{\mathrm{AA}}$, as demonstrated by Fig. 7, where the comparison between some reactance curves evaluated for both phasing line configu-

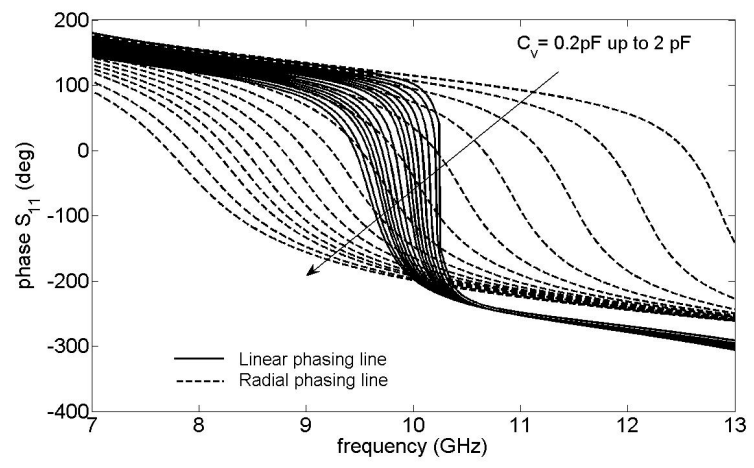

Fig. 6. Phase of coefficient $S_{11}$ vs. frequency for different diode capacitance $C_{\mathrm{v}}$ : comparison between linear and radial phasing line geometry.

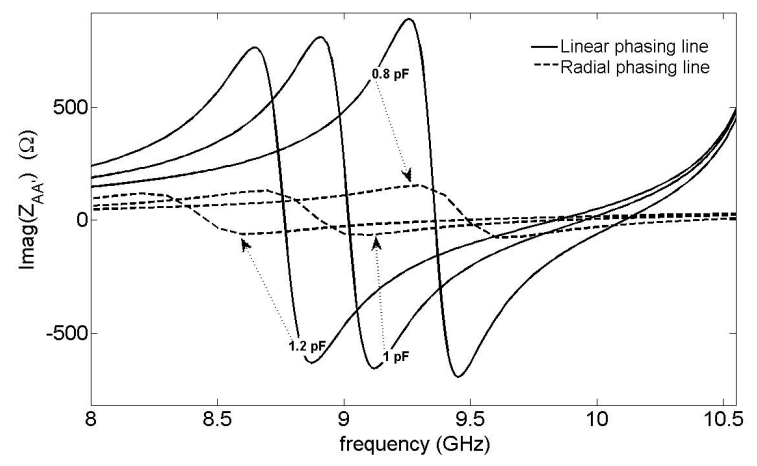

Fig. 7. Comparison between linear and radial phasing line input reactance $Z_{\mathrm{AA}}$, vs. frequency for different values of diode capacitance $C_{\mathrm{v}}$.

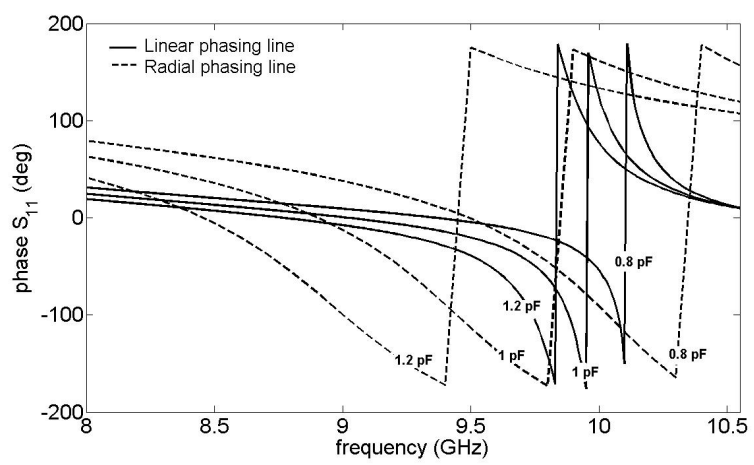

Fig. 8. Comparison between linear and radial phasing line reflection phase vs. frequency for different values of diode capacitance $C_{\mathrm{v}}$

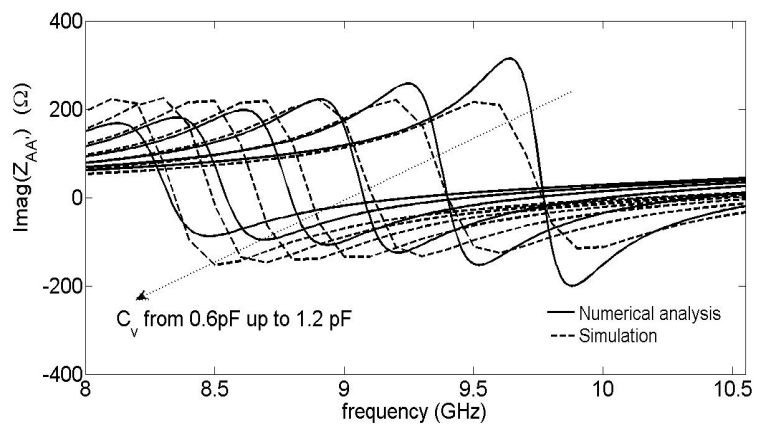

Fig. 9. Comparison between computed and simulated radial phasing line reflection phase vs. frequency for different values of diode capacitance $C_{\mathrm{v}}$. 
rations at different $C_{\mathrm{v}}$ values is reported. Furthermore, Figure 8 demonstrates how the slower frequency variations of the radial phasing line input reactance gives rise to a reflection phase curve (i.e. $\arg \left(S_{11}(f)\right)$ with a very smooth and linear behavior within the considered frequency range, which is a measure of the wideband behavior offered by the radial geometry when applied to design a varactorbased phase tuning line. In order to validate the results achieved through the implemented circuit model, an equivalent radial shaped phasing line is simulated with a fullwave commercial code. Figure 9 shows the comparison between a set of input reactances (i.e. $\left.\operatorname{Imag}\left(Z_{\mathrm{AA}^{\prime}}\right)\right)$ of the radial phasing line versus frequency, computed for different diode capacitance values with both considered numerical approaches. From the reactance curves depicted in Fig. 9, a strong similarity can be observed between fullwave simulations and equivalent circuit computations, thus confirming the validity of the proposed analysis method.

\section{Conclusion}

A wideband varactor loaded phasing line, based on the use of a couple of radial stubs, has been considered to dynamically tune the reflection phase of an aperture-coupled reflectarray cell. A simplified circuit-model approach has been adopted to perform a preliminary investigation on the improvements achievable with the radial phasing line geometry, in terms of unit cell frequency agility. The method has been fruitfully adopted to demonstrate and justify the wider band performances of radial geometry with respect to the classical linear one, when applied to design a varactor-based phase tuning line. The results achieved for a $10 \mathrm{GHz}$ unit cell demonstrate the possibility to design a frequency agile unit cell, through a proper shaping of the active tuning line, without adding any further active components, so limiting DC-biasing network complexity.

\section{References}

[1] HUANG, J., ENCINAR, J. Reflectarray Antennas. Wiley-IEEE Press, 2008. ISBN: 9780470084915.

[2] HUM, S.V., PERRUISSEAU-CARRIER, J. Reconfigurable reflectarrays and array lenses for dynamic antenna beam control: a review. IEEE Transactions on Antennas and Propagation, 2014, vol. 62, no. 1, p. 183-198. DOI: 10.1109/TAP.2013.2287296

[3] NAYERI, P., YANG, F., ELSHERBENI, A. Z. Beam-scanning reflectarray antennas: a technical overview and state of the art. IEEE Antennas and Propagation Magazine, 2015, vol. 57, no. 4, p. 32-47. DOI: 10.1109/MAP.2015.2453883

[4] GIANVITTORIO, J. P., RAHMAT-SAMII, Y. Reconfigurable patch antennas for steerable reflectarray applications. IEEE Transactions on Antennas and Propagation, 2006, vol. 54, no. 5, p. 1388-1392. DOI: 10.1109/TAP.2006.874311
[5] COSTAnzo, S., SPADAFORA, F., BORGia, A., MORENO, O.H., COSTANZO, A., DI MASSA, G. High resolution software defined radar system for target detection. Advances in Intelligent Systems and Computing, 2013, vol. 206 AISC, p. 997-1005. DOI: 10.1007/978-3-642-36981-0_94

[6] Costanzo, S., SPADAFORA, F., BORGIA, A., MORENO, H.O., COSTANZO, A., DI MASSA, G. High resolution software defined radar system for target detection. Journal of Electrical and Computer Engineering, 2013, art. no. 573217, 7 p. DOI: $10.1155 / 2013 / 573217$

[7] PERRUISSEAU-CARRIER, J. Dual-polarized and polarization flexible reflective cells with dynamic phase control. IEEE Transactions on Antennas and Propagation, 2010, vol. 58, no. 5, p. 1494-1502. DOI: 10.1109/TAP.2010.2044333

[8] GUClu, C., PERruisseaU-CARrier. J., CiVI, O. Proof of concept of a dual-band circularly-polarized RF MEMS beam switching reflectarray. IEEE Transactions on Antennas and Propagation, 2012, vol. 60, no. 11, p. 5451-5455. DOI: 10.1109/TAP.2012.2207690

[9] RODRIGO, D., JOFRE, L., PERRUISSEAU-CARRIER, J. Unit cell for frequency tunable beam scanning reflectarrays. IEEE Transactions on Antennas and Propagation, 2013, vol. 61, no. 12, p. 5992-5999. DOI: 10.1109/TAP.2013.2281375

[10] VENNERI, F., COSTANZO, S., DI MASSA, G. Reconfigurable aperture-coupled reflectarray element tuned by a single varactor diode. Electronics Letters, 2012, vol. 48, no. 2, p. 68-69. DOI: 10.1049/el.2011.3691

[11] VENNERI, F., COSTANZO, S., DI MASSA, G., BORGIA, A., CORSONELLO, P., SALZANO, M. Design of a reconfigurable reflectarray based on a varactor tuned element. In Proceedings of the 6th European Conference on Antennas and Propagation (EuCAP). Prague (CZ), 2012. DOI: 10.1109/EuCAP.2012.6206624

[12] VENNERI, F., COSTANZO, S., DI MASSA, G., MAROZZO, E., BORGIA, A., CORSONELLO, P., SALZANO, M. Beam-scanning reflectarray based on a single varactor-tuned element. International Journal of Antennas and Propagation, 2012, art. no. 290285. DOI: $10.1155 / 2012 / 290285$

[13] VenNeri, F., COSTANZO, S., DI MASSA, G. Design and validation of a reconfigurable single varactor-tuned reflectarray. IEEE Transactions on Antennas and Propagation, 2013, vol. 61, no. 2, p. 635-645. DOI: 10.1109/TAP.2012.2226229

[14] COSTANZO, S., VENNERI, F., RAFFO, A., DI MASSA, G., CORSONELLO, P. Active reflectarray element with large reconfigurability frequency range. In Proceedings of the 9th European Conference on Antennas and Propagation (EuCAP), Lisbon (Portugal), 2015.

[15] SORRENTINO, R., ROSELLI, L. A new simple and accurate formula for microstrip radial stub. IEEE Microwave and Guided Letters, 1992, vol. 2, no. 12, p. 480-482. DOI: 10.1109/75.173401

[16] COSTANZO, S., DI MASSA, G. An integrated probe for phaseless near-field measurements. Measurement: Journal of the International Measurement Confederation, 2002, vol. 31, p. 123-129. DOI: 10.1016/S0263-2241(01)00036-7

[17] GÜNEL, T., KENT, S. Numerical modeling of microstrip radial stub. Journal of Microwave Power and Electromagnetic Energy, 1997, vol. 32, p. 246-250. ISSN: 0832-7823

[18] COSTANZO, S. Synthesis of multi-step coplanar waveguide tomicrostrip transition. Progress in Electromagnetics Research, 2011, vol. 113, p. 111-126. DOI: 10.2528/PIER10112908

[19] GUPTA, K. C., GARG, R., BAHL, I., BHARTIA, P. Microstrip Lines and Slotlines. 2nd ed., rev. London (UK): Artech House, 1996. ISBN: 9780890067666. 